\title{
An Improved Image Compression Algorithm Using Wavelet and Fractional Cosine Transforms
}

\author{
Naveen kumar. $\mathbf{R}$ \\ Dept. of studies and research in Electronics, Kuvempu University, Karnataka, India \\ Email: nkr.hsd@gmail.com \\ B.N. Jagadale and J.S. Bhat \\ Dept. of studies and research in Electronics, Kuvempu University, Karnataka, India \\ Dept. of Physics, Karnataka University, Dharwad, India \\ Email: basujagadale@gmail.com, jsbhat@kud.ac.in
}

Received: 26 June 2018; Accepted: 17 September 2018; Published: 08 November 2018

\begin{abstract}
The most significant parameters of image processing are image resolution and speed of processing. Compressing the multimedia datasets, which are rich in quality and volume is challenging. Wavelet based image compression techniques are the best tools for lossless image compression, however, they suffer by low compression ratio. Conversely fractional cosine transform based compression is a lossy compression technique with less image quality. In this paper, an improved compression technique is proposed by using wavelet transform and discrete fractional cosine transform to achieve high quality of reconstruction of an image at high compression rate. The algorithm uses wavelet transform to decompose image into frequency spectrum with low and high frequency sub bands. Application of quantization process for both sub bands at two levels increases the number of zeroes, however rich zeroes from high frequency sub bands are eliminated by creating the blocks and then storing only non-zero values and kill all blocks with zero values to form reduced array. The arithmetic coding method is used to encode the sub bands. The Experimental results of proposed method are compared with its primitive two dimensional fractional cosine and fractional Fourier compression algorithms and some significant improvements can be observed in peak signal to noise ratio and self-similarity index mode at high compression ratio.
\end{abstract}

Index Terms-Discrete Wavelet Transform (DWT) decomposition, One-dimensional Discrete Fractional Cosine Transform (DFrCT), Quantization.

\section{INTRODUCTION}

Image compression is a technique employed for reducing the number of bits to represent an image data, which in turn reduces the storage space [1-3]. The compression methods minimize the size of an image by eliminating the redundancy. In a lossy compression method, strict elimination of all redundant information leads to a loss in image quality [4,5], whereas, in lossless image compression some of the redundancy, which is very significant for image quality is retained and encoded to increase the quality of image [6,7]. In transform based image compression, a set of transformed coefficients are generated by applying defined filters. Eliminating the correlated coefficients or zero coefficients based on defined threshold leads to compression $[8,9]$.

The discrete cosine transform (DCT) is well suited for block-based lossy compression but a large number of blocks leads to a problem of artifact and low compression performance [10]. The wavelet-based compression technique is the best choice for lossless compression, amongst all transforms due to its multi-resolution property [11]. The wavelet domain has the key role in feature extraction by identifying the smoothness in different functional spaces and represent them in terms of wavelet coefficients $[12,13]$. On the other hand, Discrete fractional Cosine transform (DFrCT) has the close relation with DCT with one free parameter (fractional order) as its function. The normal DCT is achieved by reducing the free parameter to zero, otherwise, it will be a conservative DCT, and hence use of optimum fractional order gives us perfect compression ratio [14]. Several wavelets based hybrid lossy compression techniques are used to improve the signal quality at high compression rate. A hybrid method of singular value decomposition (SVD) and embedded zero tree wavelet (EZW) for ECG signal [15], shows significant improvement in compression ratio and excellent quality of image reconstruction but has complexity in extracting the features. A lossy compression [16], using Wavelet difference reduction(WDR) with SVD is used to get highquality reconstruction. Similarly, a hybrid wavelet compression algorithm [17] uses DWT for decomposition and two level DCT to compress low-frequency wavelet coefficients resulting in high compression ratio. Thus, compressing low-frequency wavelet coefficients without disturbing the compactness is the key to achieve good compression $[18,19]$. In this paper, we present an improved grayscale image compression technique using DWT and DFrCT.

The paper is organized as follows: In sections 2-4 a concise preface to DWT, DFrCT and Quantization for 
compression are presented. The proposed compression scheme using DWT-DFrCT is presented in section 5. Section 6 gives the results and discussions, followed by conclusion in section 7 .

\section{DISCRETE WAVELET TRANSFORM FOR DECOMPOSITION}

The wavelet-based signal analysis has become more and more popular because of its multiresolution property. This property is exploited in converting the original signal into its frequency spectrum. In wavelet decomposition process, an input signal is transformed into fine discrete samples of inherent mother wavelet functions or wavelet coefficients of different resolutions [20]. For each decomposition level, it uses scaling and mother wavelet function to generate two classes of wavelet coefficients (approximate and detailed coefficients). In two-dimensional wavelet decomposition, for each level it produces approximate (LL), horizontal detail (HL), vertical detail(LH) and diagonal detail $(\mathrm{HH})$ sub bands as shown in Fig.1.

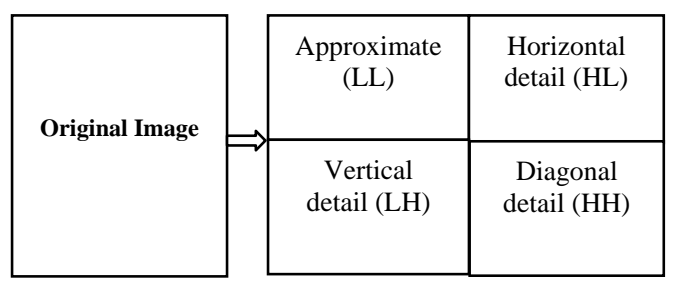

Fig.1. Wavelet decomposition.

As decomposition level increases, the image undergoes partition of four sub-image and significant information spread towards the top left corner and residual information concentration spreads towards a right bottom corner of the image [21]. Hence, by neglecting the right bottom sub band the redundancy in an image can be decreased and it helps in the compression process.

\section{ONE Dimensional DisCRETE FRACTIONAL COSINE TRANSFORM:}

The Discrete fractional Cosine Transform (DFrCT) is the general version of discrete cosine transform (DCT) with one additional free parameter as a fractional order. Fractional order modulates the transform into DCT or conventional DCT. In this work one dimensional DCT-1 kernel is derived by using $\mathrm{N}$ point DFT Eigen vectors then convert it into DFrCT kernel.

Consider,

$V=\left[V_{0}, V_{1}, \ldots \ldots \ldots, V_{N-2}, V_{N-1}, V_{N-2}, \ldots \ldots V_{1}\right]^{T}$ is an even eigen vectors of (2N-2)- point DFT kernel matrix, $F_{2 N-2} V=\lambda V(\lambda=1,-1)$

Where,

$$
\hat{V}=\left[V_{0}, \sqrt{2} V_{1}, \ldots, \sqrt{2} V_{N-1}, V_{N-1}\right]^{T}
$$

will be the eigen vectors of $\mathrm{N}$ - Point DCT kernel matrix, where $\lambda$ are its eigen vectors

$$
C_{N}^{I} \hat{V}=\lambda \hat{V}
$$

In the computation of DCT, the infinite number of eigen vectors are generated from even Hermite-Gauss eigenvectors of Fourier matrix [23]. However, for DFrCT kernel matrix the eigenvector $\hat{V}_{N}$ have the eigen values of $e^{-j n \alpha}$ with 'n' being even ( $\left.\alpha=\pi / 2\right)$. Hence DFrCT kernel is defined for $\mathrm{N}$ point as,

$$
\begin{aligned}
& C_{N, \alpha}=\hat{V}_{N} \hat{D}_{N}^{2 \alpha / \pi} \hat{V}_{N}^{t}
\end{aligned}
$$

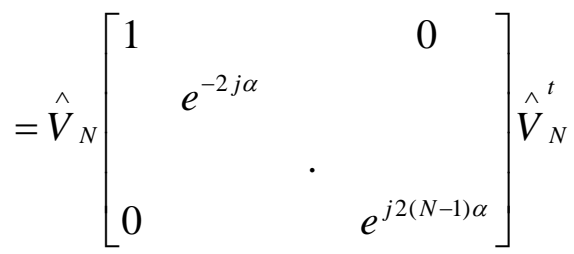

where $\hat{V}_{N}=\left[\hat{V}_{0},\left|\hat{V}_{2}\right| \ldots \ldots \ldots . . . \mid \hat{V}_{2 N-2}\right], \hat{V}_{N}$ is an eigenvector for DFrCT derived from the $\mathrm{N}^{\text {th }}$ order DFT Hermite Eigen vectors from [24]. From angular rotation parameter $\alpha=\pi / 2$, we can derive DFrCT, for $\alpha=1$, it will become DFT kernel and $C_{N, \alpha}$ becoming identity matrix.

In general, for image compression, two-dimensional $\mathrm{DFrCT}$ is used by processing row and column, but in this paper, we have used one-dimensional DFrCT to code the wavelet decomposed LL sub bands. Here each column of LL sub band has been compressed with an optimized angle of rotation $a$.

\section{QUANTIZATION}

The quantization is applied to both LL and non-LL sub-bands individually in two stages. In the first stage, multiply the quantization scale with the median value of sub band to increase the correlation by using equation (6) then divide the wavelet decomposed sub bands by quantization scale to reduce the redundancy.

$$
\begin{gathered}
Q_{1}=\text { Quantization_Scale } \times \max \left(s u b_{-} \text {band_A }\right) \\
S u b_{-} \text {band_A }=\operatorname{round}\left(\operatorname{sub} b_{-} \text {band } / Q_{1}\right)
\end{gathered}
$$

Level two quantization is performed for only LL sub bands by creating the quantization matrix and transformed coefficients are divided by Q2, which eliminates the insignificant coefficients by inserting zeros[25]. 


$$
Q_{2}(m, n)=\left\{\begin{array}{l}
1, \text { if }(m=1, n=1) \\
m+n+R, \text { if }(m \neq 1, n \neq 1)
\end{array}\right.
$$

In this paper, the quantization scale for LL sub band is fixed to 0.01 , but for now, LL sub bands are varied in accordance with compression ratio.

\section{PRoposed COMPRESSION SCHEME}

The wavelet transform facilitates analysis of the signal in time-frequency domain by representing the source image into a cluster of significant coefficients in four frequency spectrums. A compact time domain analysis in DFrCT helps us to code the more significant sub bands with reduced size without reducing the signal quality. This combination is more efficient because it generates more de-correlated coefficients than spatial based compression algorithms. Fig.2. shows the algorithm for the proposed method of compression.

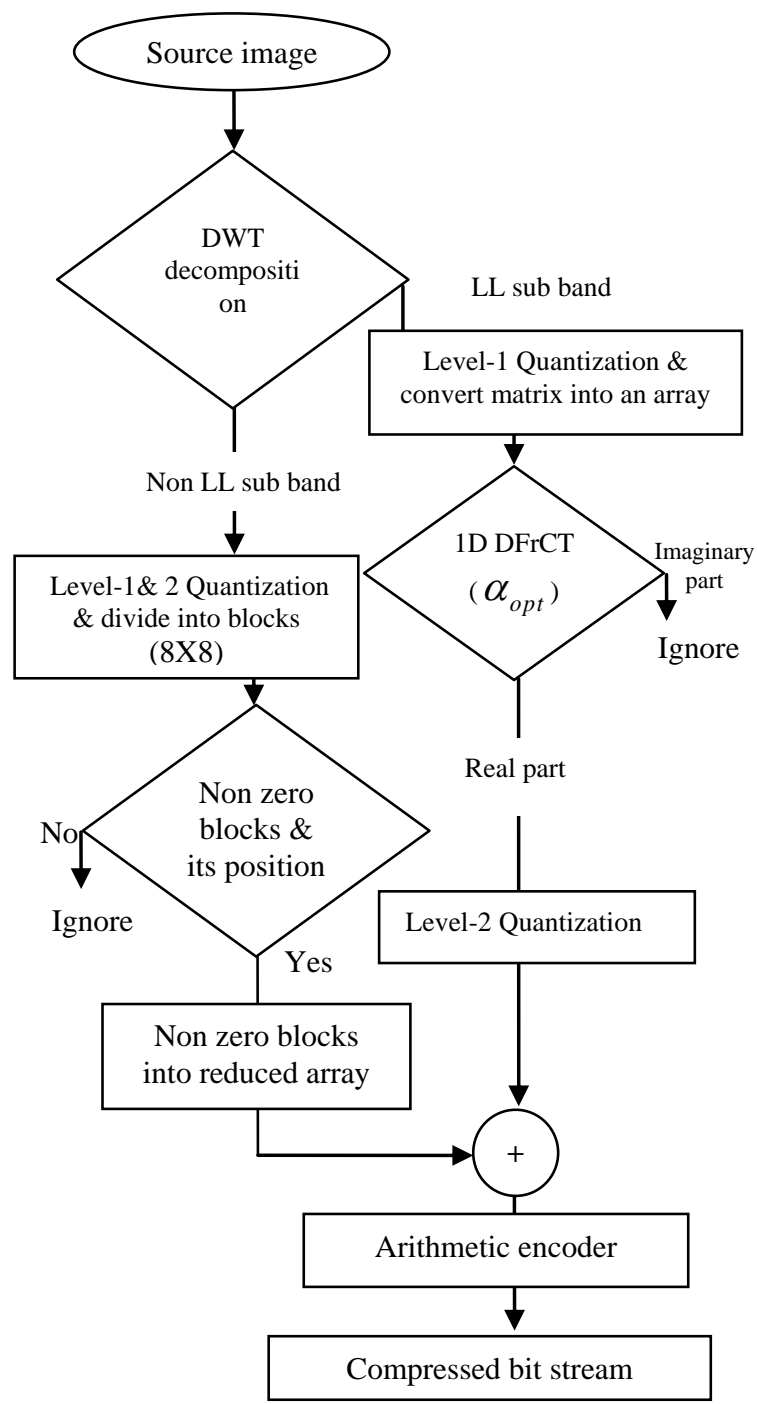

Fig.2. Pipelined view of a proposed lossy compression algorithm.
This compression algorithm uses fallowing steps,

Step1: The image is subjected to wavelet decomposition with the sufficient number of levels, which split the image into LL and non-LL sub bands. First level non-LL sub bands are neglected. Then apply the level-1 quantization for both the sub bands.

Step2: The non-LL sub bands are segmented into blocks of size (8x8). Store the significant non-zero blocks and its position and the remaining blocks are zero. Apply leve- 2 quantization and then encode them by arithmetic coding.

Step3: Create the level-2 quantization matrix for each column of LL sub band. Create N-point DFrCT kernel by optimized rotational angle $\alpha$ following the steps are given below:

i. Generate $\mathrm{M}_{\mathrm{P}}$ point $\mathrm{DFT}$ Hermite even eigen vectors $M_{P}=2(N-1)$.

ii. By using equation (2) compute DCT_I eigen vectors from DFT Hermite even eigen vectors.

iii. Then find out DFrCT kernel by using equation (3).

Imaginary part is ignored in encoding gives lossy compression. The optimum fractional order used for different test images for good compression, and store them as a reduced array followed by the arithmetic encoder with header tags.

Step4:. IDFrCT is applied for decoded LL sub band and decoded blocks of nonzero non-LL sub bands are restored to their original position remaining all blocks are padded by zero. This is orthogonal process hence the decompression is inverse of the compression method. Finally, IDWT is applied for both sub bands to reconstruct high-quality source image.

The compression percentage (CP) and peak signal to noise ratio (PSNR) are calculated with respect to the original image and compressed image. The increase in PSNR values indicates better reconstruction quality of images.

$$
\left[C P=\frac{\left[I_{1}(m, n)-I_{2}(m, n)\right]}{I_{2}(m, n)} \times 100\right]
$$

where, mean square error (MSE) is given as,

$$
\begin{aligned}
& M S E=\frac{\sum_{M, N}\left[I_{1}(m, n)-I_{2}(m, n)\right]^{2}}{M \times N} \\
& P S N R=10 \log _{10}\left(\frac{255 \times 255}{M S E}\right)
\end{aligned}
$$

where, $I_{1}$ in $I_{2}$ are original and reconstructed images, respectively, with $m, n$ representing row and columns. 
In this work, the PSNR values are enhanced by computing DWT-DFrCT and IDWT-IDFrCT for LL sub bands repeatedly with optimized quantization factor and fractional orders $\left(a_{\alpha}\right)$. The non-LL sub bands are compressed by erasing the more number of zero blocks in sub bands with high quantization factors. At the same time, LL sub bands are compactly coded and compressed with an optimal fractional order $\left(\mathrm{a}_{\alpha}\right)$.

\section{EXPERIMENTAL RESULTS AND DISCUSSION:}

The strength of proposed compression algorithm is evaluated by numerical simulations. The original test images 'Lena', 'Barbara', 'Cameraman', 'Rice', and 'IC' dimension $512 \times 512$ are used to analyze the compression techniques.

This proposed compression method uses three optimization measures to increase the compression performance.

\section{A. Use of mother wavelet for decomposition}

At first, the 'Debauchees-4' (Db-4) mother wavelet is used for decomposition. Its quadrature mirror filter coefficients for decomposition are given as:

Table.1 Db4 wavelet coefficients used for decomposition

\begin{tabular}{|l|l|l|l|}
\hline \multicolumn{2}{|c|}{ HPF coefficients } & \multicolumn{2}{c|}{ LPF coefficients } \\
\hline h0= & 0.0105974018 & $\mathrm{~g} 0=$ & -0.2303778133 \\
$\mathrm{~h} 1=$ & 0.0328830116 & $\mathrm{~g} 1=$ & 0.71484657055 \\
$\mathrm{~h} 2=$ & 0.0308413818 & $\mathrm{~g} 2=$ & -0.6308807679 \\
$\mathrm{~h} 3=$ & -0.187034811 & $\mathrm{~g} 3=$ & -0.0279837694 \\
$\mathrm{~h} 4=$ & -0.027983769 & $\mathrm{~g} 4=$ & 0.1870348117 \\
$\mathrm{~h} 5=$ & 0.6308807679 & $\mathrm{~g} 5=$ & 0.0308413818 \\
$\mathrm{~h} 6=$ & 0.7148465705 & $\mathrm{~g} 6=$ & -0.0328830117 \\
$\mathrm{~h} 7=$ & 0.2303778133 & $\mathrm{~g} 7=$ & -0.0105974018 \\
\hline
\end{tabular}

Two vanishing moments in $\mathrm{Db} 4$ helps us to reconstruct the original signal from half of its wavelet coefficients (smoothing the functions of original signal). However, this property of $\mathrm{Db} 4$ helps in neglecting the level one detail coefficients, during reconstruction. As long as we increase the decomposition level, the low frequency sub band becomes more correlated. But, levels are limited to three for this discussion, since levels above this limit leads to a loss in reconstructed image quality.

\section{B. Optimization in DFrCT}

The LL sub band obtained from wavelet decomposition has more number of correlation coefficients. It is essential to optimize DFrCT kernel with specific fractional order for LL sub band coding. Use of Eigen vector based DFrCT computational procedure is more preferable due to its flexibility over the selection of fractional order in coding chirp signal. In this discussion, LL sub band of Lena image is used for the optimization study of fractional orders in DFrCT. The Fig..3 shows the 131 samples of LL sub band coefficients of 'Lena' image are compressed by using DFrCT kernel with fractional order $\left(a_{\alpha}\right) 0.97$ for $80 \%$ CP. From this figure one can observe that original samples are coded with few samples of DFrCT coefficients.

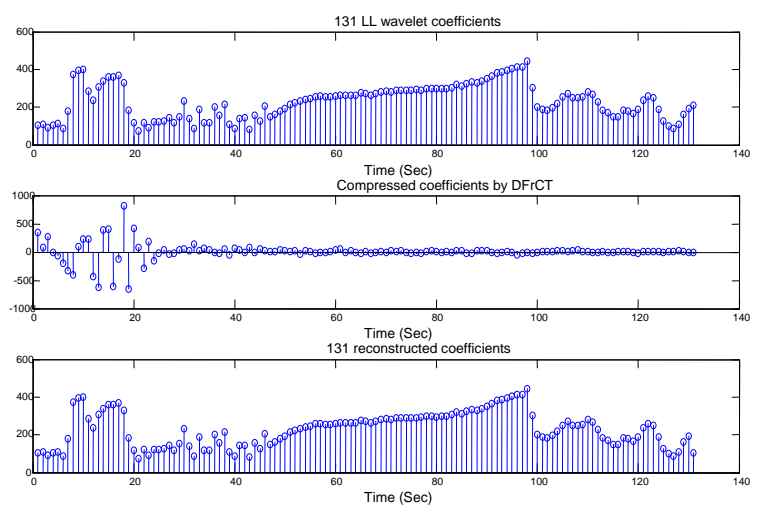

Fig.3. Compression and reconstruction of 131 wavelet coefficient using $\operatorname{DFrCT}\left(\mathrm{a}_{\alpha}=0.97\right)$ at $80 \% \mathrm{CP}$.

The detailed coefficient values obtained from DFrCT itself has imaginary value as well as real values in the complex number. But, this algorithm involves in calculations of only real part and neglect the imaginary part of the complex number in order to boost the image compression.

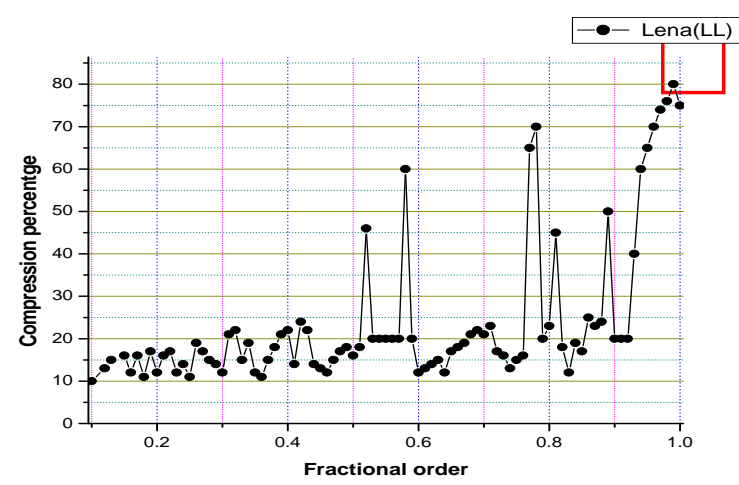

Fig.4. Fractional orders manually computed for different CP by using LL sub band of 'Lena' image.

In this algorithm fractional order $\left(a_{\alpha}\right)$ of DFrCT is optimized with respect to $\mathrm{CP}$. Thus, fractional orders are manually calculated and select the one specific value where maximum $\mathrm{CP}$ is obtained. From Fig.4., it is observed that the $\mathrm{CP}$ is not so linear with respect to fractional order and it is gradually increasing above the 0.70 up to 0.99 fractional orders (*highlighted in red box in Fig.4. has maximum CP). Hence, this range of $\left(a_{\alpha}\right)$ is used throughout this compression process.

\section{Optimization in Quantization and coding of non $L L$ sub band}

This algorithm uses two levels of quantization. Compressed LL coefficients are very sensitive in nature, which needs less quantization scale and hence we use 
fixed scale of 0.01 throughout the algorithm. However, non LL sub bands has high redundancy and needs coarse quantization, hence we used variable scales ranging from 0.001 to 0.2 . Defining quantization scale is directly proportional to $\mathrm{CP}$ of the algorithm. Increase in quantization value reduces the size of compressed bit stream with small compromise with quality of image. The Fig.6. Reveals the graphical comparison of a three non LL sub bands of Lena image are quantized with different quantization scales over $\mathrm{CP}$.

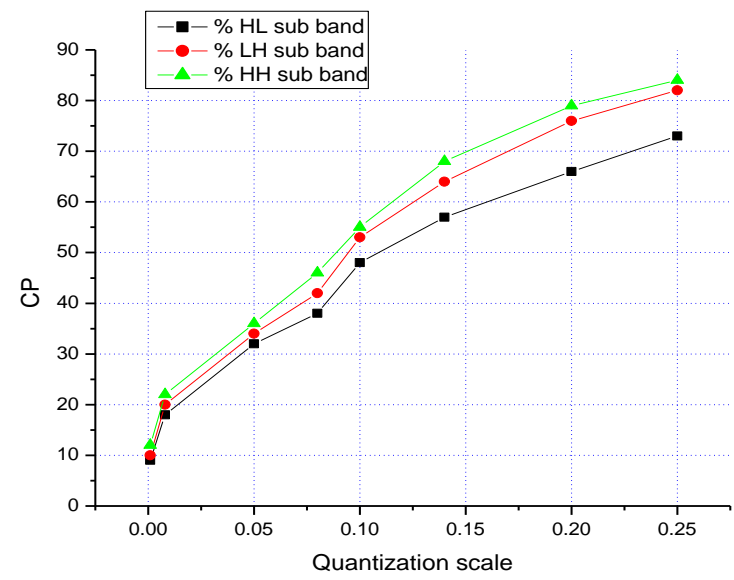

Fig.5. Quantization scale versus CP for non LL sub band.
This graph proves that use of one specific quantization scale is enough for compression of both three sub band.

After quantization process, the non LL sub band enriched by large number of zeroes and they are eliminated by creating the blocks. The number of non zero blocks identified in non LL sub bands are tabulated in T able.1.

Table.2.number of non-zero blocks identified in quantized non LL sub bands (128X128) of 'Lena' image.

\begin{tabular}{|l|c|c|c|c|}
\hline \multirow{2}{*}{$\begin{array}{l}\text { Block } \\
\text { size }\end{array}$} & \multirow{2}{*}{$\begin{array}{c}\text { Total } \\
\text { blocks }\end{array}$} & \multicolumn{3}{|c|}{ No. non zero blocks } \\
\cline { 3 - 5 } & & HL & LH & HH \\
\hline 8 X8 & $\mathbf{2 5 6}$ & $\mathbf{2 1 9}$ & $\mathbf{1 8 7}$ & $\mathbf{1 4 8}$ \\
\hline 16X16 & 64 & 52 & 48 & 33 \\
\hline $32 \times 32$ & 16 & 15 & 13 & 12 \\
\hline 64X64 & 4 & 4 & 4 & 4 \\
\hline
\end{tabular}

From above table we found that increase in block size increases the non-zero blocks and forced to code as reduced array. Even one non-zero value exist in a block, is considered as non-zero block and it must encode. Hence in this algorithm $8 \times 8$ blocks is used to enhance the image compression.

Table 3. MSE and PSNR values obtained with the proposed method for different compression percentage for Lena, Barbara, cameramen and IC images.

\begin{tabular}{|c|c|c|c|c|c|c|}
\hline Test images & $\begin{array}{c}\text { Compression } \\
\text { percentage }(\%)\end{array}$ & $\begin{array}{c}\text { Wavelet } \\
\text { decomposition level }\end{array}$ & $\begin{array}{l}\text { Quantization factor } \\
\text { for non-LL }\end{array}$ & $\left(a_{\alpha}\right)$ for $L L$ & MSE & PSNR \\
\hline \multirow{6}{*}{ Lena } & 20 & 1 & 0.001 & 0.93 & 18.745 & 36.23 \\
\hline & 40 & 3 & 0.002 & 0.90 & 20.189 & 35.29 \\
\hline & 50 & 3 & 0.006 & 0.84 & 20.221 & 35.19 \\
\hline & 60 & 3 & 0.01 & 0.99 & 20.175 & 35.08 \\
\hline & 70 & 3 & 0.03 & 0.98 & 21.194 & 34.86 \\
\hline & 80 & 3 & 0.08 & 0.97 & 26.154 & 33.95 \\
\hline \multirow{6}{*}{ Barbara } & 20 & 1 & 0.002 & 0.98 & 146.87 & 28.76 \\
\hline & 40 & 3 & 0.01 & 0.97 & 170.98 & 25.89 \\
\hline & 50 & 3 & 0.012 & 0.94 & 171.08 & 25.82 \\
\hline & 60 & 3 & 0.015 & 0.92 & 175.26 & 25.79 \\
\hline & 70 & 3 & 0.02 & 0.99 & 179.54 & 25.78 \\
\hline & 80 & 3 & 0.03 & 0.97 & 183.4 & 25.22 \\
\hline \multirow{6}{*}{ Cameramen } & 20 & 1 & 0.001 & 0.91 & 12.79 & 37.09 \\
\hline & 40 & 3 & 0.008 & 0.94 & 12.74 & 37.06 \\
\hline & 50 & 3 & 0.01 & 0.99 & 13.08 & 36.94 \\
\hline & 60 & 3 & 0.01 & 0.95 & 13.23 & 36.59 \\
\hline & 70 & 3 & 0.02 & 0.98 & 14.45 & 36.01 \\
\hline & 80 & 3 & 0.02 & 0.96 & 15.04 & 35.96 \\
\hline \multirow{6}{*}{ Rice } & 20 & 1 & 0.01 & 0.92 & 44.38 & 31.96 \\
\hline & 40 & 3 & 0.1 & 0.96 & 63.19 & 30.12 \\
\hline & 50 & 3 & 0.15 & 0.98 & 73.60 & 29.86 \\
\hline & 60 & 3 & 0.2 & 0.97 & 87.50 & 28.71 \\
\hline & 70 & 3 & 0.25 & 0.98 & 103.51 & 27.98 \\
\hline & 80 & 3 & 0.35 & 0.99 & 132.4 & 26.38 \\
\hline \multirow{6}{*}{ IC } & 20 & 1 & 0.02 & 0.97 & 98.78 & 29.07 \\
\hline & 40 & 3 & 0.02 & 0.95 & 101.68 & 28.05 \\
\hline & 50 & 3 & 0.05 & 0.96 & 105.12 & 27.91 \\
\hline & 60 & 3 & 0.09 & 0.98 & 116.37 & 27.42 \\
\hline & 70 & 3 & 0.15 & 0.94 & 148.96 & 27.08 \\
\hline & 80 & 3 & 0.2 & 0.97 & 159.74 & 25.97 \\
\hline
\end{tabular}


The above optimization steps are included in proposed compression algorithm and results are calculated as follows: The Table .3 shows the proposed compression algorithm at CP 20, 40, 50, 60, 70 and $80 \%$ by varying the wavelet decomposition levels, optimum quantization scale and fractional order $\left(a_{\alpha}\right)$ are used. Here we found that the $20 \% \mathrm{CP}$ is achieved by wavelet decomposition at level one and anything above 20 is achieved at level three decomposition. At the same time, the quantization scale for non-LL sub-band varies from 0.001 to 0.2 and for LL sub band compression by using optimum fractional order varies from 0 to 1 . The simulated results for Lena image at different CP is shown in Fig.6. It is observed that the proposed algorithm preserves quality of reconstructed image even at CP $85 \%$. This quality loss in reconstructed image at $85 \% \mathrm{CP}$ is not so observable for normal eye perception.

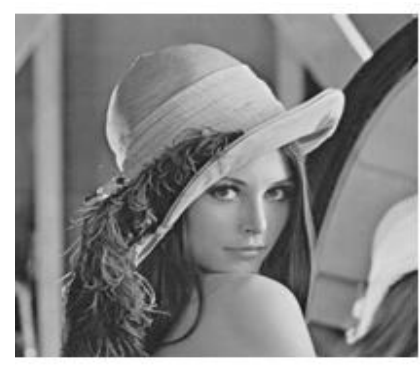

(a)

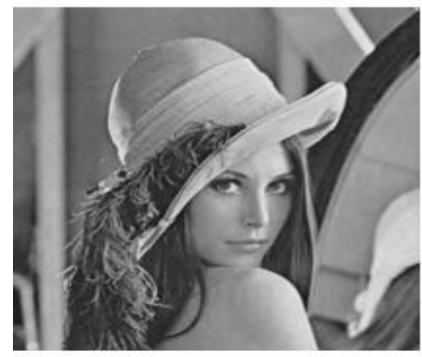

(d)

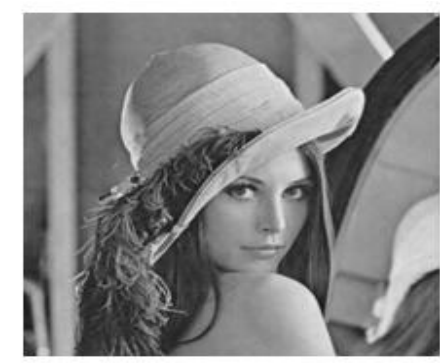

(b)

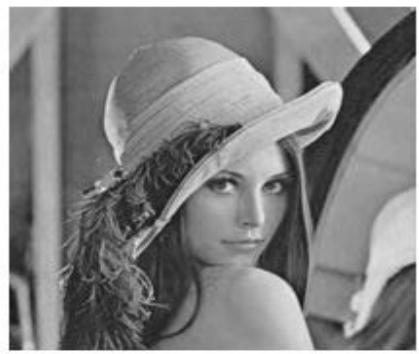

(e)

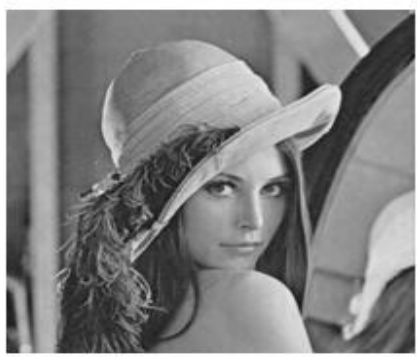

(c)

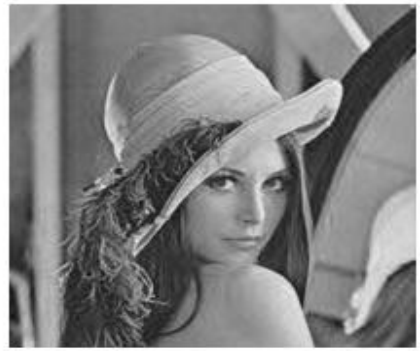

(f)

Fig.6. (a)Original Lena image(512X512) and compressed by using proposed method at(b) $20 \%$ with PSNR 36.23db, (c) $40 \%$ with PSNR 35.29db, (d) $60 \%$ with PSNR $35.08 \mathrm{db}$, (e) $80 \%$ with PSNR $33.29 \mathrm{db}$ and (f) $85 \%$ with PSNR $32.33 \mathrm{db}$.

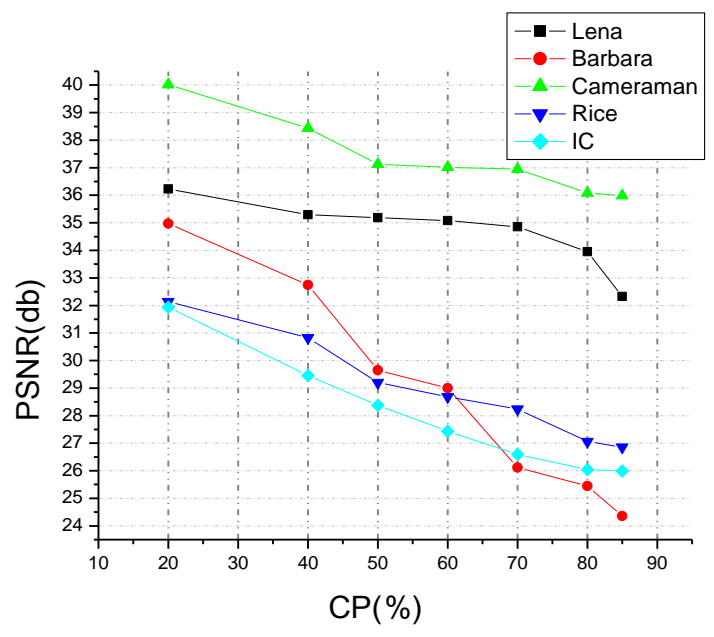

Fig.7. PSNR for different images plotted as the function of compression percentage obtained using DWT-DFrCT.
The Fig.7 shows there is an existence of ambiguity in between PSNR and CP for different test images. Whereas we observe that, increase in CP leads to a reduction in PSNR values and vice-versa.

Here two comparison study is carried out with proposed method for compete of interest. At first, proposed method is compared with two dimensional DFrCT and DFrFT. Secondly, with current compression technology JPEG and JPEG2000. In Tabel.4. the results obtained with a proposed method, DFrFT and DFrCT [26] are tabulated. The PSNR, MSE values are fixed at CP of $75 \%$. We find that the reconstruction quality of Lena and cameraman is better than the other methods. Because, these image samples have rich inter pixel relations and contain less edge information. Fig. 8 shows the graphical PSNR comparison of the proposed method using DFrFT and DFrCT at compression percentage $75 \%$. The results show that quality of reconstruction by proposed methods is significantly better in terms of PSNR. 
Table 4. Comparison of MSE and PSNR values, obtained for different images, using proposed method with DFrCT and DFrFT

\begin{tabular}{|c|c|c|c|c|c|c|c|c|c|c|c|}
\hline \multirow[t]{2}{*}{ Test images } & \multirow{2}{*}{$\begin{array}{c}\text { Compression } \\
\text { percentage }\end{array}$} & \multicolumn{3}{|c|}{ DFrCT[26] } & \multicolumn{3}{|c|}{ DFrFT [26] } & \multicolumn{4}{|c|}{$\begin{array}{l}\text { DWT-DFrCT } \\
\text { (level 3) }\end{array}$} \\
\hline & & $\mathrm{a}_{\alpha}$ & MSE & PSNR & $\mathrm{a}_{\alpha}$ & MSE & PSNR & $\mathrm{Q}$ & $\mathrm{a}_{\alpha}$ & MSE & PSNR \\
\hline Lena & 75 & 0.94 & 313.8 & 22.0 & 0.93 & 97.8 & 25.21 & 0.05 & 0.94 & 22.73 & 34.52 \\
\hline Barbara & 75 & 0.99 & 543.04 & 19.6 & 0.91 & 339.7 & 20.91 & 0.04 & 0.98 & 172.8 & 25.73 \\
\hline Cameramen & 75 & 0.99 & 464.30 & 20.37 & 0.97 & 180.7 & 24.40 & 0.02 & 0.96 & 14.28 & 36.54 \\
\hline Rice & 75 & 0.92 & 161.52 & 24.95 & 0.92 & 122.2 & 24.57 & 0.3 & 0.98 & 121.4 & 27.28 \\
\hline IC & 75 & 0.99 & 512.26 & 19.94 & 0.94 & 44.8 & 26.11 & 0.2 & 0.96 & 153.6 & 26.26 \\
\hline
\end{tabular}

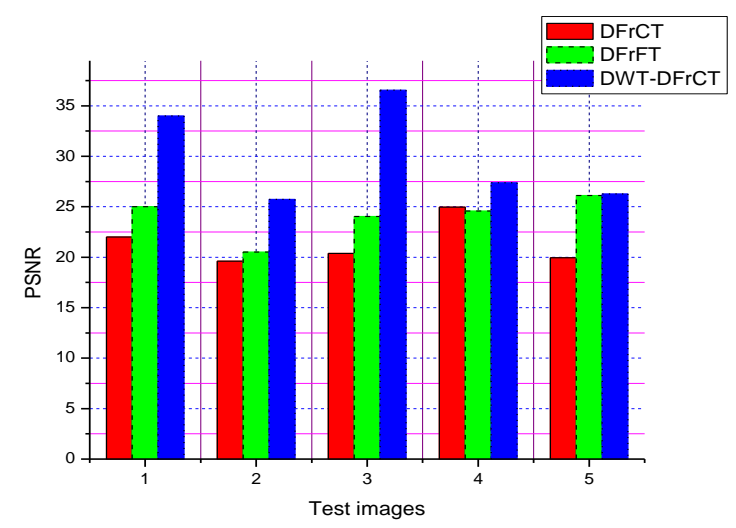

Fig.8. Comparison of PSNR for test images (1.Lena; 2.Barbara;

3.cameraman; 4.rice; 5.IC) using DFrCT, DFrFT and DWT-DFrCT.
The comparison study extends in Table.5 of proposed method with JPEG and JPEG2000. However some images like, Barbara and IC has less PSNR value than JPEG2000, but better than JPEG. These two images have rich edge information, which needs fine refining for each set of wavelet coefficients.

From above simulation and comparison study we noticed that the images with less edge information are recovered better by proposed method with reduced error. If image has more number of edges, then the performance of this compression algorithm is going to be reduced. This is due to DFrCT coding with irrespective of all edge coefficients and two levels of quantization process kills few coefficients at encoding stage.

Table 5. Comparison of MSE and PSNR values, obtained for different images, using proposed method with JPEG and JPEG2000

\begin{tabular}{|c|c|c|c|c|c|c|c|c|c|}
\hline \multirow[t]{2}{*}{ Test images } & \multirow{2}{*}{$\begin{array}{c}\text { Compression } \\
\text { percentage }\end{array}$} & \multicolumn{2}{|c|}{ JPEG } & \multicolumn{2}{|c|}{ JPEG2000 } & \multicolumn{4}{|c|}{$\begin{array}{c}\text { DWT-DFrCT } \\
\text { (level 3) }\end{array}$} \\
\hline & & MSE & PSNR & MSE & PSNR & $\mathrm{Q}$ & $\mathrm{a}_{\alpha}$ & MSE & PSNR \\
\hline Lena & 80 & 25.33 & 28.67 & 24.04 & 33.46 & 0.05 & 0.94 & 23.44 & 33.52 \\
\hline Barbara & 80 & 178.9 & 24.33 & 183.49 & 31.24 & 0.04 & 0.98 & 178.9 & 24.33 \\
\hline Cameramen & 80 & 18.06 & 34.11 & 22.76 & 36.07 & 0.02 & 0.96 & 15.99 & 35.65 \\
\hline Rice & 80 & 131.4 & 26.02 & 132.8 & 26.05 & 0.3 & 0.98 & 132. & 26.71 \\
\hline $\mathrm{IC}$ & 80 & 172.0 & 26.76 & 186.0 & 30.07 & 0.2 & 0.96 & 160.0 & 25.54 \\
\hline
\end{tabular}

This proposed algorithm is target to increase the quality of image reconstruction at high $\mathrm{CP}$. Hence it is quite obvious to compromise with PSNR values to implement high $\mathrm{CP}$.

\section{CONCLUSIONS}

In this paper, a new image compression algorithm based on wavelet and fractional transform to improve the compression quality have been proposed with three key features to enhance compression efficiency. First, selection of wavelet decomposition level with suitable mother wavelet, the work uses db5 mother wavelet and levels are limited to one and three. Secondly, the sensitive LL sub bands compressed and encoded by the application of one-dimensional DFrCT with an optimal fractionalorder. Thirdly, detailed (non-LL) sub bands are compressed by suitable quantization scale and only nonzero blocks in a sub band are stored. Except less coding efficiency in edge reconstruction, this algorithm competes with current compression methods. This proposed algorithm is suitable for applications, which requires high compression percentage and bit compromised with PSNR. Overall simulation results and comparative studies show better performance with high compression percentages.

\section{REFERENCES:}

[1] B.S.Manjunath, W.Y.Ma, "Texture Features for Browsing and Retrieval of image data", IEEE Trans. on pattern analysis and machine intelligence, Vol.18, No 8, pp. 837842, 1996.

[2] k. Sayood, Introduction to data compression, $3^{\text {rd }}$ ed., academic press, Morgan Kaufmann Publishers, 2006

[3] W.B.Pennebaker, J.L. Mitchell, JPEG still image data compression standard, $1^{\text {st }}$ ed., Kluwer Academic Publishers, 1992.

[4] T.L.B.yng, B.G.Lee, H.Yoo, "A low complexity lossless frame memory compression for display device", IEEE Trans. on Consumer Electronics, Vol. 54, No.3, pp. 14531458, 2008.

[5] H. You, J. M. Jo, J. C. Jeong, “A hierarchical lossless 
image compression based on modified Hadamard transform", in Proceedings of the $10^{\text {th }}$ Workshop on Image processing and Understanding, pp. 305-310,1998.

[6] Jia Li, "An improved wavelet image lossless compression algorithm", Journal of Optik, Vol.124, pp:1041$1044,2013$.

[7] Macarena Boix, Begona canto, "Wavelet transform application to the compression of images", Journal of Mathematical and computer modeling, Vol-52, pp.12651270, 2010.

[8] R.D. Dony, S. Haykin, "Optimally adaptive transform coding", IEEE Trans. Image Processing , Vol.10, pp. 1358-1370,1995.

[9] A.K. Jain, Fundamentals of Digital Image Processing, Prentice-Hall, Englewood Cliffs, NJ, 1989, p. 569.

[10] Tsai. M and Hung. H, K., "DCT and DWT Based Image Watermarking Using Sub-Sampling", Proceeding of the Fourth International Conference on Machine Learning and Cybern, Guangzhou, pp. 5308-5313, 2005.

[11] J.W. Woods, S.D. O'Neil, "Subband coding of Images", IEEE Trans. Acoustic Speech Signal Processing, ASSP34, pp. 1278-1288,1986.

[12] Ronald.A.de vore, BjornJawerth, Bradly.J.Lucier, "Image compression through wavelet coding", IEEE Trans. on information theory, Vol. 38, No. 2,pp.719-746, 1992.

[13] KUMAR, Naveen; JAGADALE, B. N.; BHAT, J. S.. "Improved Binary Tree Coding for Image Compression using Modified Singular Value Decomposition". Journal of Informatics and Mathematical Sciences, [S.1.], v. 10, n. 1-2, p. 109 - 118, aug. 2018.

[14] C.Vijaya, J.S.Bhat, "Signal compression using discrete fractional Fourier transform and set partitioning in the hierarchical tree", Journal of Signal Processing, Vol. 86, pp.1976-1983.2008.

[15] Ranjeeth Kumar, A. Kumar, G. K.singh, "Hybrid method based on singular value decomposition and embedded zero tree wavelet technique for ECG signal compression", Journal of Computer methods and programs in biomedicine, Vol. 129, pp. 135-148,2016.

[16] A. M. Rufai, Golamreza. A Hasndemirel, "Lossy image compression using singular value decomposition and Wavelet difference reduction", Journal of Digital signal processing. Vol.24, pp.117-123, 2014.

[17] M.M.Siddeq, M.A.Rodrigues, "A Novel 2D Image compression algorithm based on two level DWT and DCT Transforms with an enhanced minimization-matrix-size algorithm for high resolution structured light 3D surface reconstruction", Journal of 3DR Express, Springer pub., pp:6-26.2015.

[18] Tim Bruylants, Adrian Munteanu, Peter Schelkens, "Wavelet-based volumetric medical image compression", Journal of Signal Processing: Image Communication, Vol.31, pp. 112-133.2015.

[19] A.S. Poulakidas, A. Srinivasan, O. E. gecioglu, O. Ibarra, T. Yang, "Image compression for fast wavelet-based subregion retrieval", Journal Theoretical computer science, Vol. 240, pp:447-469,2000.

[20] Strang. G and Nguyen.T, Wavelets and Filter Banks, Wellesley-Cambridge Press, Wellesley, MA, 1996, HTTP: //www-math. mit. edu/gs/book/wfb.html.

[21] R. C. Gonzalez and R. E. Woods, Digital Image Processing, Addison Wesley Publishing Company, Reading, 2001.

[22] Z. Wang, "Fast algorithm for the discrete W transform and for the discrete Fourier transform," IEEE Trans. Acoustic, Speech Signal Processing, vol. ASSP-32, pp.
803-816, 1984

[23] Yeh, M.H. Pei, S.C, "A method for the discrete fractional Fourier transform computation". IEEE Trans. Signal Process. Vol. 51, No. 3, pp. 889-891, 2003.

[24] Narsimha, M.J, Peterson, A.M, "On the computation of the discrete cosine transform", IEEE Trans. communication, Vol. 26, No. 6, pp.934-936, 1978.

[25] Devid Solomon, Data compression, The complete reference, 4th ed. Springer Verlag London Limited-2007.

[26] Singh. K,(2006), "Performance of discrete fractional Fourier transform classes in signal processing applications (Doctoral Thesis), from:dspace.http://thapar.edu: 8080 /jspui/bitstream/123456789/94/1/P92233.pdf.

\section{Authors' Profiles}

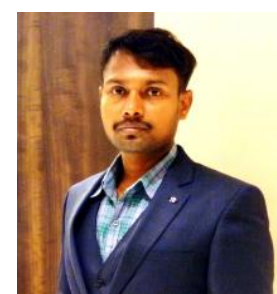

Dr. Naveen Kumar.R, he was born on 31 august 1987. He received his $\mathrm{PhD}$ and masters degree in Digital signal processing from Kuvempu University, Karnataka, India, in 2018 and 2010 respectively. Currently he is perusing his Post doctoral fellow in Medical Electronics from Karnataka University, Karnataka, India. His research interests are signal/image processing, VLSI, Embedded systems, model-tech simulations, FPGA, Biomedical instrumentations, Parallel computations, etc.

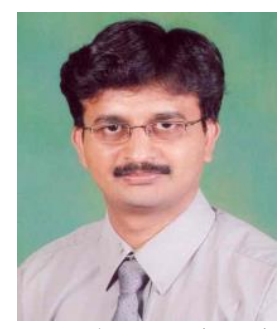

Dr. Basavaraj N. Jagadale. is an assistant professor in kuvempu university, India. He received his Post Doctoral fellow in study of biomedical imaging from Pennsylvania University, Upenn, USA. He also received his $\mathrm{PhD}$ and masters degree in Electronics from Karnatak University. He is member of BOE and BOS in Kuvempu university, Bangalore university, Mangalore university. His research interests are signal/image processing, $\mathrm{C}$ in data structure, model-tech simulations, FPGA, UNIX based designing, etc. He published more than 50 research articles, 2 book chapters in various field of engineering.

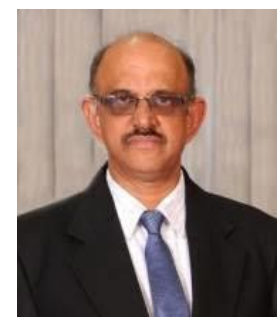

Prof. J.D. Bhat, is Director in IIT, surat ,India. He served as administrative head as Registrar evolution, head of examination boards, chairman of Physics dept. etc in Karnatak Univerisy, Karnataka, India. He is member of BOE and BOS in karntak university, Kuvempu university, Bangalore university, Mangalore university. $\mathrm{He}$ received his $\mathrm{PhD}$ and masters degree in Physics from Karnatak University, karnataka, India. His research interests are timefrequency analysis of signals, Digital signal/image processing, microwave engineering, design of high speed computational devices, Antenna designing, Nano technology etc. He is a reviewer of 10 reputed Elsevier Journals, published more than 160 research articles in various field of applications, and completed 2 UGC major projects. 
How to cite this paper: Naveen kumar. R, B.N. Jagadale, J.S. Bhat, "An Improved Image Compression Algorithm Using Wavelet and Fractional Cosine Transforms", International Journal of Image, Graphics and Signal Processing(IJIGSP), Vol.10, No.11, pp. 19-27, 2018.DOI: 10.5815/ijigsp.2018.11.03 